\title{
Improving User Experience of Virtual Health Assistants: Scoping Review
}

Rachel G Curtis, BPsych (Hons), PhD; Bethany Bartel, BA; Ty Ferguson, BPhysio (Hons); Henry T Blake, BHmMvt, BHlthSc (Hons); Celine Northcott, BBus, MRsch; Rosa Virgara, BPhysio (Hons); Carol A Maher, BPhysio (Hons), $\mathrm{PhD}$

UniSA Allied Health and Human Performance, Alliance for Research in Exercise, Nutrition and Activity, University of South Australia, Adelaide, Australia

\section{Corresponding Author:}

Rachel G Curtis, BPsych (Hons), PhD

UniSA Allied Health and Human Performance

Alliance for Research in Exercise, Nutrition and Activity

University of South Australia

GPO Box 2471

Adelaide, 5001

Australia

Phone: 61883022455

Email: Rachel.Curtis@unisa.edu.au

\section{Abstract}

Background: Virtual assistants can be used to deliver innovative health programs that provide appealing, personalized, and convenient health advice and support at scale and low cost. Design characteristics that influence the look and feel of the virtual assistant, such as visual appearance or language features, may significantly influence users' experience and engagement with the assistant.

Objective: This scoping review aims to provide an overview of the experimental research examining how design characteristics of virtual health assistants affect user experience, summarize research findings of experimental research examining how design characteristics of virtual health assistants affect user experience, and provide recommendations for the design of virtual health assistants if sufficient evidence exists.

Methods: We searched 5 electronic databases (Web of Science, MEDLINE, Embase, PsycINFO, and ACM Digital Library) to identify the studies that used an experimental design to compare the effects of design characteristics between 2 or more versions of an interactive virtual health assistant on user experience among adults. Data were synthesized descriptively. Health domains, design characteristics, and outcomes were categorized, and descriptive statistics were used to summarize the body of research. Results for each study were categorized as positive, negative, or no effect, and a matrix of the design characteristics and outcome categories was constructed to summarize the findings.

Results: The database searches identified 6879 articles after the removal of duplicates. We included 48 articles representing 45 unique studies in the review. The most common health domains were mental health and physical activity. Studies most commonly examined design characteristics in the categories of visual design or conversational style and relational behavior and assessed outcomes in the categories of personality, satisfaction, relationship, or use intention. Over half of the design characteristics were examined by only 1 study. Results suggest that empathy and relational behavior and self-disclosure are related to more positive user experience. Results also suggest that if a human-like avatar is used, realistic rendering and medical attire may potentially be related to more positive user experience; however, more research is needed to confirm this.

Conclusions: There is a growing body of scientific evidence examining the impact of virtual health assistants' design characteristics on user experience. Taken together, data suggest that the look and feel of a virtual health assistant does affect user experience. Virtual health assistants that show empathy, display nonverbal relational behaviors, and disclose personal information about themselves achieve better user experience. At present, the evidence base is broad, and the studies are typically small in scale and highly heterogeneous. Further research, particularly using longitudinal research designs with repeated user interactions, is needed to inform the optimal design of virtual health assistants.

(J Med Internet Res 2021;23(12):e31737) doi: 10.2196/31737 


\section{KEYWORDS}

virtual assistant; conversational agent; chatbot; eHealth; digital health; design; user experience; mobile phone

\section{Introduction}

\section{Background}

Advancements in machine learning and artificial intelligence offer promise for delivering automated, tailored, convenient health assistance with an unprecedented level of sophistication and personalization and are already contributing to the transformation of health care [1]. Virtual assistants can be broadly defined as digital services designed to simulate human conversation and provide personalized responses based on input from the user. They can be programmed with structured conversations or to answer the user's questions. Capabilities range from simple menu or multiple choice-based assistants to more sophisticated virtual assistants with natural language processing that recognize free speech or text. At present, virtual assistants are widely deployed in web-based banking and service settings, reducing reliance on staff by being available to answer consumers' questions about products and services on demand. Virtual assistants are also increasingly being designed for various health applications, such as delivering cognitive behavior therapy for depression and anxiety [2], improving diet and physical activity [3], and conducting remote patient monitoring [4]. Despite the exciting potential for using virtual assistants for health purposes, the use of virtual assistants in health could be ineffective or even have unintended negative consequences if the technology does not meet the user's needs and preferences.

The user experience of a virtual health assistant can be defined as the user's perceptions and responses (eg, emotions, beliefs, preferences, and behaviors) that result from its use or anticipated use [5]. User experience is influenced by a range of factors, including presentation, functionality, and interactive behavior [5]. It is important to optimize the design of virtual assistants to provide a positive user experience and promote engagement. A growing body of evidence suggests that design characteristics that influence the look and feel of the virtual assistant, such as visual appearance, communication method, and language features, are an important consideration for design, as such design characteristics can significantly influence users' psychological and emotional responses and engagement with technology-based applications [6,7]. In addition, although some design decisions may not affect the cost (eg, whether an avatar should be male or female), other decisions may have a major impact on the cost of designing a virtual health assistant (eg, whether an avatar should be animated with facial expressions). Understanding how such design characteristics influence user experience will assist in using finite health software development budgets most effectively.

Previous literature has proposed general guidelines for designing voice user interfaces [8] and accessible conversational user interfaces for different disability groups [9], as well as virtual assistants for specific purposes such as teaching [10] and in-vehicle assistance [11]. Optimal design techniques are likely to depend on the purpose of the virtual assistant $[12,13]$; therefore, recommendations specifically in the context of health are needed. Although research has examined methods of assessing the usability of virtual assistants in the health domain [14], clear guidelines on maximizing the user experience of virtual health assistants are lacking.

An important first step toward constructing guidelines for the development of virtual health assistants was achieved by the literature review conducted by ter Stal et al [15] in 2018, which aimed to identify the researched design characteristics for embodied conversational agents (virtual assistants that have an animated avatar) in health. The review provided a comprehensive overview of the existing literature, with results suggesting that speech and/or textual output and facial and gaze expressions were the most commonly researched design characteristics. The secondary aims of ter Stal et al [15] were to identify the outcome variables used in the research and the effects of the design characteristics. The authors concluded that, based on the immature body of evidence at the time, there was no consensus on the optimal design characteristics for embodied conversational agents in health. Results highlighted key avenues for future research, including the fact that more research is needed on all design characteristics to advance the field. Notably, the review by ter Stal et al [15] included studies using any research design and studies where participants viewed stimuli but did not necessarily interact with a virtual assistant.

\section{Objectives}

The evidence base for the use of interactive virtual health assistants is rapidly growing in both size and quality. In particular, experimental research designs with interactive virtual assistants are being reported increasingly, which should provide clearer evidence of the influence of design characteristics on user experience. A scoping review methodology offers an explicit, systematic means to overview this large and diverse body of literature using rigorous methods to minimize bias [16]. In this study, we seek to undertake the first scoping review of design characteristics of virtual health assistants, with a view to bring together the strongest evidence available regarding the effects of design characteristics on the user experience of interactive virtual health assistants. In particular, the aims of our scoping review are as follows:

1. Provide an overview of all the experimental research examining how design characteristics of virtual health assistants affect user experience

2. Summarize research findings of experimental research examining how design characteristics of virtual health assistants affect user experience

3. Identify whether research supports making recommendations for the design of virtual health assistants

Bringing together the available evidence on how design characteristics affect the user experience of virtual health assistants will assist researchers and software developers in making decisions about the look and feel of their software and developing the most user-friendly and effective virtual health assistants. 


\section{Methods}

This review is reported according to the PRISMA-ScR (Preferred Reporting Items for Systematic Reviews and Meta-Analyses Extension for Scoping Reviews) checklist [17].

\section{Eligibility Criteria}

Eligibility criteria were designed using the population, intervention, comparator, and outcome framework (population: adults; intervention: virtual health assistant; comparator: design characteristics; and outcome: user experience) [18]. Original research articles in peer-reviewed journals and full-length conference papers were included.

\section{Population}

Studies with adult samples (aged $\geq 18$ years) were included.

\section{Intervention}

Studies examining virtual health assistants were included. For this review, we considered virtual health assistants to be any virtual assistant aimed at the health consumer (general population or patient) relating to the prevention, management, or treatment of any physical or mental health condition, as well as clinical research. Virtual health assistants were included if they functioned on any electronic device (eg, smartphone, computer, and headset). Wizard of $\mathrm{Oz}$ virtual assistants (where the user believes they are interacting with a computer-automated virtual assistant, but the virtual assistant is operated by a human [19]) were included.

\section{Comparator}

Studies comparing design characteristics between $\geq 2$ versions of a virtual health assistant were included. For this review, we defined design characteristics as characteristics of the virtual assistant that influence its look and feel without affecting its core content, purpose, or function. Examples of design characteristics include visual cues such as whether the virtual health assistant has an avatar (ie, an image that represents the virtual assistant), language style, and interaction modality (ie, text or speech). Between- and within-subject experimental designs were included.

\section{Outcome}

Studies evaluating user experience outcomes were included. For this review, we defined user experience to include self-reported evaluations of the virtual assistant or the user's interaction with the virtual assistant that indicated a more positive or negative experience (eg, trustworthiness, likeability, enjoyment, and ease of use), affect, intentions to continue using the virtual assistant, and objective measures of user engagement (eg, frequency, duration, or nature of the interaction with the virtual health assistant). Only quantitative data were included.

\section{Exclusion Criteria}

Dissertations, review articles, conference abstracts, and studies with children were excluded. Virtual assistants used for training or educating medical professionals, as well as robots with a physical body, were excluded. Studies were excluded if participants did not interact with the virtual health assistant; that is, they did not provide any input into the system. Studies were also excluded if the virtual health assistant was not the main component of the health program. Studies were excluded if they evaluated only 1 version of a virtual assistant (ie, nonexperimental research design with no comparator) or if they compared a virtual assistant to a human. Dependent variables that were not associated with a more positive or negative user experience-for example, those used as manipulation checks (eg, where participants were asked to confirm whether a realistic-looking assistant was indeed more realistic looking than a cartoon-style assistant)—were excluded.

\section{Information Sources and Search Strategy}

A cross-disciplinary search of the literature was conducted on June 4, 2020, and included 5 electronic databases across the fields of health and information technology: Web of Science, MEDLINE, Embase, PsycINFO, and ACM Digital Library. Search terms for virtual assistant AND design characteristics were included in the search strategy (Table 1). Eligibility specifying the virtual assistant related to health, user experience outcomes, and experimental study design was assessed at screening. Searches were limited to the English language with no limit on publication date. Reference lists of the included studies and other key papers in the field were searched to identify further studies (pearling).

Table 1. Search terms.

\begin{tabular}{|c|c|}
\hline Search category & Search terms \\
\hline Virtual assistant & $\begin{array}{l}\text { “conversational agent*” OR “conversational system*” OR “dialog system*” OR “dialogue system*” OR “assistance } \\
\text { technolog*” OR “relational agent*” OR “virtual agent*” OR “virtual assistant*” OR “embodied agent*” OR chatbot* }\end{array}$ \\
\hline Design characteristics & 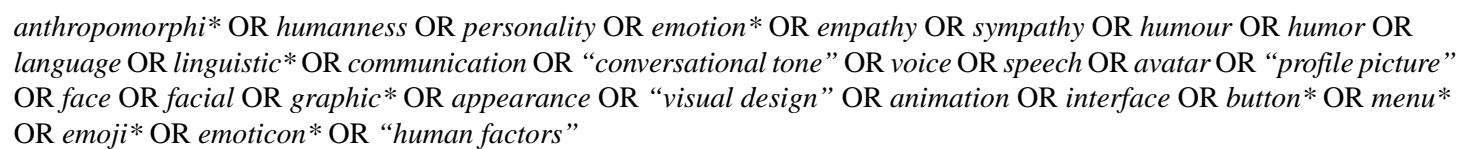 \\
\hline
\end{tabular}

\section{Evidence Selection and Data Charting}

Search results from each database were imported into EndNote (Clarivate) [20], in which duplicates were removed. Studies were screened based on title and abstract. Studies that met the eligibility criteria progressed to full-text screening. The full texts of the studies were then screened to determine final eligibility. Articles were screened by 1 of 2 raters. Raters screened a randomly generated selection of 20 articles in duplicate, and the agreement was $100 \%$. A custom form was developed and used for data charting (Multimedia Appendix 1). Extracted data included population, sample size, age, gender, study country, cultural background, health domain, purpose of the virtual assistant, name of the virtual assistant, Wizard of $\mathrm{Oz}$ 
design, device used, animated character, output modality, input modality, whether the interaction was scripted (whether participants were told what to say), duration of interaction, experimental design, and study results. If articles included multiple studies, data extraction was completed only for studies meeting the eligibility criteria. Where multiple eligible studies were included in an article, data were extracted separately. Where relevant outcomes were measured but not compared statistically between experimental conditions, authors were contacted to provide additional information.

\section{Data Synthesis}

Study characteristics were compiled for all the studies included in the review. Where a study was reported in multiple articles, articles were compiled as 1 study with a primary reference indicated, as well as an indication of additional references. To facilitate data synthesis across diverse research designs, overarching categories were constructed to describe the health domains, design characteristics, and outcomes. Retrospective thematic analysis was used to identify similar health domains, design characteristics, and outcomes to construct the relevant categories. After data extraction was completed, lists of all reported health domains, design characteristics, and outcomes were compiled. After familiarization with the data, the first author sorted them into similar categories using an inductive approach (ie, directed by the data with no preconceived categories). These categories were reviewed with the senior author, refined, and named.

Data were synthesized descriptively. Descriptive statistics were used to summarize the body of research. A matrix of the design characteristics and outcome categories was constructed to summarize the research findings. Results in the matrix were based on statistical results reported in the articles. Where interactions were examined (eg, in factorial designs or examining interactions with participant characteristics), main effects were included in the matrix. Studies could report results for 1 or multiple outcomes within a particular outcome category.
Results were categorized as positive, negative, or no effect. Where studies reported multiple results in a single outcome category, they were categorized as positive if all multiple outcomes showed positive effects, mixed positive if multiple outcomes were reported with both positive and nonsignificant effects, negative if all multiple outcomes showed negative effects, mixed negative if multiple outcomes were reported with both negative and nonsignificant effects, and no effect if multiple outcomes showed no significant effects.

Authors from 2 studies provided additional data on measures that were not compared between experimental groups. Independent sample $t$ tests (2-tailed) were conducted, and the results were included in the matrix. In total, 4 studies did not present a statistical analysis comparing relevant experimental conditions; therefore, these studies are included only in the text description.

\section{Results}

\section{Overview}

The search identified 6879 articles after duplicates were removed. Of the 6879 articles, 6763 (98.31\%) were deemed ineligible based on title and abstract screening. We identified 30 additional records through reference lists. In total, 146 articles $(116 / 6879,1.69 \%$ from the database search plus 30 from reference lists) were screened at full text. Of the 146 articles, $98(67.1 \%)$ were deemed ineligible; $81(55.5 \%)$ did not examine an interactive virtual health assistant, $8(5.5 \%)$ did not compare design features between $\geq 2$ virtual health assistants, 4 (2.7\%) did not report user experience outcomes, 2 (1.4\%) were not adult samples, $1(0.7 \%)$ did not report original research, $1(0.7 \%)$ was not a journal of conference paper, and $1(0.7 \%)$ did not have the virtual health assistant as a main component of the program. Of the 146 articles, a final 48 (32.9\%) articles were included in the scoping review (Figure 1). From the 48 articles, 45 unique studies were identified (5 studies were reported in multiple articles, whereas 3 articles contained multiple studies). 
Figure 1. PRISMA (Preferred Reporting Items for Systematic Reviews and Meta-Analyses) flowchart. VHA: virtual health assistant.

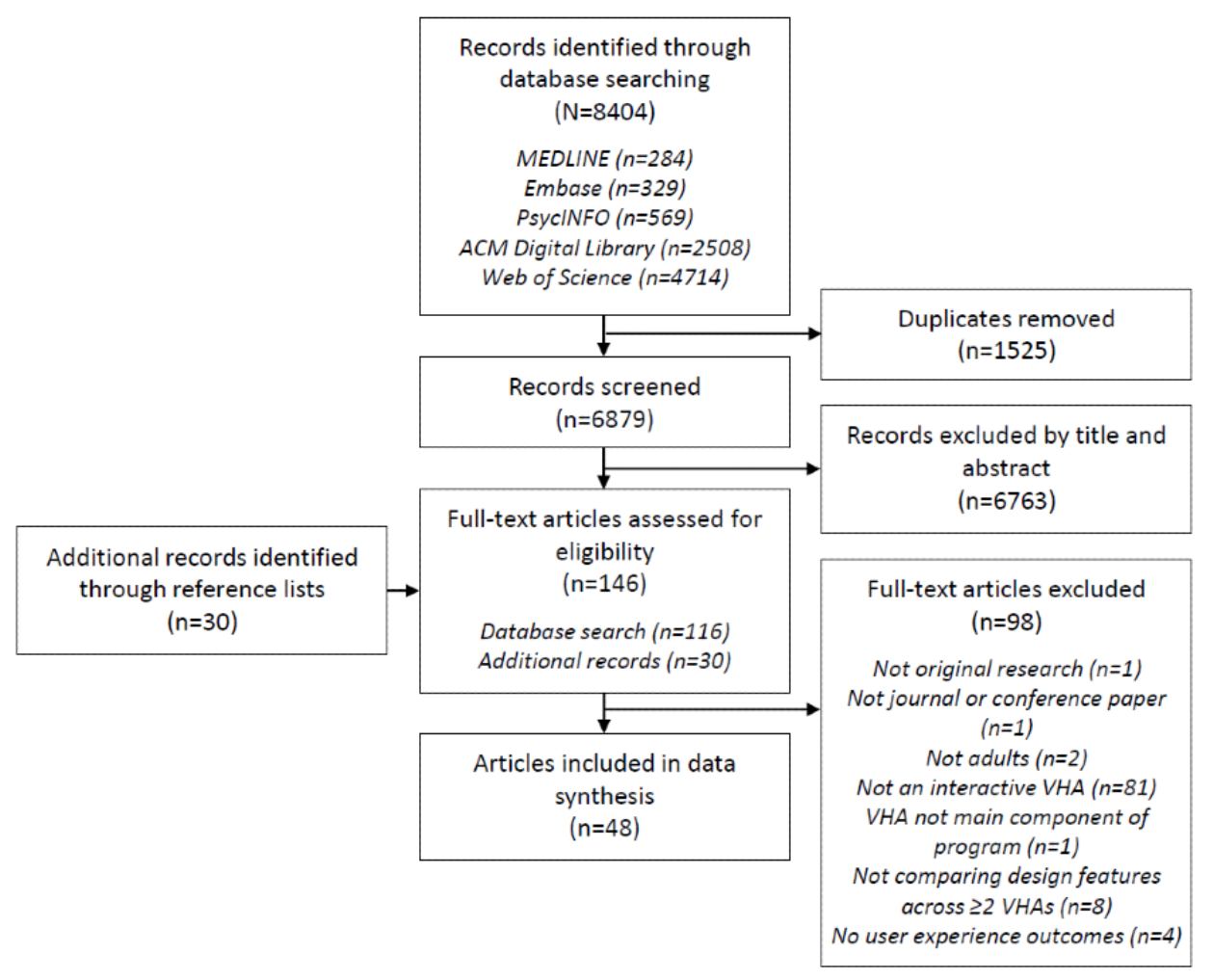

Multimedia Appendix 2 [21-68] provides an overview of the participant characteristics and study designs for all studies included in the review. Table 2 summarizes the characteristics of the body of research. The virtual assistants used in the research were categorized into 8 health domains: physical activity (aimed to increase exercise), nutrition (aimed to improve diet), alcohol consumption (aimed to reduce alcohol consumption), mental health (eg, aimed to improve mood), medical information or treatment (eg, discussed colorectal cancer screening), sexual health (eg, provided advice about sexually transmitted infections), multiple health behaviors (eg, aimed to improve both exercise and diet), and other (eg, aimed to prevent carpal tunnel). A total of 27 design characteristics were examined in the literature. These were categorized into 5 categories: visual design (eg, realism, age, and body shape of an animated avatar), interface design (eg, input modality), conversational style and relational behavior (eg, empathy and relational behavior and personality), combined visual and conversational design (eg, variability in language and background scene assessed simultaneously), and cultural and organizational affiliation (eg, culturally tailored argumentation and appearance; see Table 3 for the full list of design characteristics). We identified 140 outcome variables, which were categorized into nine categories: virtual assistant personality traits (eg, credible and intelligent), relationship (eg, intimacy and relationship closeness), ease of use (eg, cognitive load and ease of use), satisfaction (eg, enjoyment, satisfaction, and usefulness), emotion (eg, positive and negative affect), use intention (eg, intention to keep using the virtual assistant), engagement (eg, interaction duration), and disclosure (eg, self-disclosure detail and intimacy; see Multimedia Appendix 3 for a full list of outcomes by category). Outcome assessment most frequently used Likert scales, with server logs and conversation transcripts used to assess engagement and disclosure.

Most studies were conducted in the United States, with a greater number of studies conducted during more recent years (ie, between 2017 and 2020). Several authors led multiple studies (ie, Bickmore [25-27,29,31], Creed [34,35], Olaffsson, [53,54], Ring [56-58], and Zhou [67,68]). Most studies examined conversational style and relational behavior or visual design and assessed outcomes in the categories of personality, satisfaction, relationship, and use intention. Virtual assistants most frequently related to mental health and physical activity. Those addressing multiple health behaviors frequently examined physical health and nutrition together. Most virtual assistants had an animated avatar and used speech output and multiple-choice input. Most virtual assistants were automated (did not use a WizardofOz design), and participant input was not scripted. Studies were most frequently conducted with between 21 and 100 participants in a single session using a between-subjects design, where participants were allocated to evaluate 1 version of the virtual assistant. Participants were most frequently from the general population, with a larger proportion of females than males. Studies were most often published in conference proceedings in fields related to interdisciplinary research on intelligent virtual agents and human-computer interactions, with fewer published in health-related fields. 
Table 2. Summary of study characteristics $(\mathrm{N}=45)$.

\begin{tabular}{|c|c|}
\hline Study Characteristics & Value, $\mathrm{n}(\%)$ \\
\hline \multicolumn{2}{|l|}{ Year } \\
\hline $2017-2020$ & $18(40)$ \\
\hline 2013-2016 & $11(24)$ \\
\hline 2009-2012 & $10(22)$ \\
\hline 2005-2008 & $6(13)$ \\
\hline \multicolumn{2}{|l|}{ Country } \\
\hline United States & $25(56)$ \\
\hline United Kingdom & $4(9)$ \\
\hline Other & $7(16)$ \\
\hline Not available & $9(20)$ \\
\hline \multicolumn{2}{|l|}{ Sample size } \\
\hline $1-20$ & $6(13)$ \\
\hline $21-50$ & $16(36)$ \\
\hline $51-100$ & $14(31)$ \\
\hline $101-200$ & $5(11)$ \\
\hline $201-500$ & $4(9)$ \\
\hline \multicolumn{2}{|l|}{ Duration } \\
\hline Single session & $37(82)$ \\
\hline Multiple sessions & $8(18)$ \\
\hline \multicolumn{2}{|l|}{ Health domain } \\
\hline Mental health & $11(24)$ \\
\hline Physical activity & $10(22)$ \\
\hline Multiple health behaviors & $7(16)$ \\
\hline Medical information or treatment & $6(13)$ \\
\hline Nutrition & $4(9)$ \\
\hline Sexual health & $2(4)$ \\
\hline Alcohol consumption & $1(2)$ \\
\hline Other & $4(9)$ \\
\hline \multicolumn{2}{|l|}{ Design category $^{\mathbf{a}}$} \\
\hline Conversational style and relational behavior & $22(49)$ \\
\hline Visual design & $12(27)$ \\
\hline Interface design & $6(13)$ \\
\hline Cultural and organizational affiliation & $5(11)$ \\
\hline Combined visual and conversational design & $2(4)$ \\
\hline \multicolumn{2}{|l|}{ Outcome category } \\
\hline Personality & $30(67)$ \\
\hline Satisfaction & $20(44)$ \\
\hline Relationship & $19(42)$ \\
\hline Use intention & $17(38)$ \\
\hline Engagement & $11(24)$ \\
\hline Ease of use & $8(18)$ \\
\hline Emotion & $7(16)$ \\
\hline
\end{tabular}




\begin{tabular}{|c|c|}
\hline Study Characteristics & Value, $\mathrm{n}(\%)$ \\
\hline Disclosure & $5(11)$ \\
\hline \multicolumn{2}{|c|}{ Virtual assistant characteristics } \\
\hline \multicolumn{2}{|l|}{ Animated avatar } \\
\hline Yes $^{\mathrm{b}}$ & $31(69)$ \\
\hline No & $14(31)$ \\
\hline \multicolumn{2}{|l|}{ Output } \\
\hline Speech $^{\mathrm{c}}$ & $33(73)$ \\
\hline Text & $11(24)$ \\
\hline Not available & $1(2)$ \\
\hline \multicolumn{2}{|l|}{ Input } \\
\hline Multiple choice & $26(58)$ \\
\hline Speech $^{\mathrm{c}}$ & $11(24)$ \\
\hline Text & $7(16)$ \\
\hline Not available & $1(2)$ \\
\hline \multicolumn{2}{|l|}{ Wizard of $\mathrm{Oz}$} \\
\hline No & $37(82)$ \\
\hline Yes & $8(18)$ \\
\hline \multicolumn{2}{|l|}{ Scripted } \\
\hline No & $41(91)$ \\
\hline Yes & $4(9)$ \\
\hline
\end{tabular}

${ }^{\mathrm{a}} \mathrm{N}$ sums to $>45$ studies and $100 \%$ because 2 studies examined design characteristics in multiple categories.

${ }^{\mathrm{b}}$ Includes studies where at least one experimental condition used an animated avatar.

${ }^{\mathrm{c}}$ Includes studies where at least one experimental condition used speech. 
Table 3. Summary of research findings ( $\mathrm{N}=41)$.

\begin{tabular}{|c|c|c|c|c|c|c|c|c|c|}
\hline \multirow{2}{*}{$\begin{array}{l}\text { Design character- } \\
\text { istics }\end{array}$} & \multirow{2}{*}{$\begin{array}{l}\text { Values, } \\
\mathrm{n}(\%)\end{array}$} & \multicolumn{8}{|c|}{ Outcomes (effect) } \\
\hline & & Personality & Relationship & Ease of use & Satisfaction & Emotion & Use intention & Engagement & Disclosure \\
\hline \multicolumn{10}{|l|}{ Visual design } \\
\hline $\begin{array}{l}\text { Animated } \\
\text { avatar (vs no } \\
\text { visual repre- } \\
\text { sentation) }\end{array}$ & $3(7)$ & 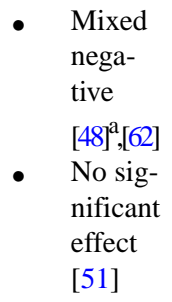 & $\begin{array}{l}\text { Mixed nega- } \\
\text { tive } \\
{[48]^{\mathrm{a}},[62]}\end{array}$ & $\begin{array}{l}\text { No signifi- } \\
\text { cant effect } \\
{[48]^{\mathrm{a}}}\end{array}$ & $\begin{array}{l}\text { No signifi- } \\
\text { cant effect } \\
{[48]^{\mathrm{a}},[51,62]}\end{array}$ & $\begin{array}{l}\text { No signifi- } \\
\text { cant effect } \\
{[48]^{\mathrm{a}},[51]}\end{array}$ & $\begin{array}{l}\text { No significant } \\
\text { effect }[48]^{\mathrm{a}}\end{array}$ & $-^{b}$ & - \\
\hline $\begin{array}{l}\text { Realistic (vs } \\
\text { cartoon) }\end{array}$ & $4(10)$ & $\begin{array}{ll}- & \begin{array}{l}\text { Posi- } \\
\text { tive }\end{array} \\
& {[64]} \\
- & \text { Mixed } \\
\text { nega- } \\
\text { tive } \\
\text { [57] } \\
\text { No sig- } \\
\text { nificant } \\
\text { effect } \\
{[57,62]}\end{array}$ & $\begin{array}{l}\text { No signifi- } \\
\text { cant effect } \\
{[62]}\end{array}$ & $\begin{array}{l}\text { No signifi- } \\
\text { cant effect } \\
{[64]}\end{array}$ & $\begin{array}{ll}\text { - } & \text { Mixed } \\
\text { positive } \\
\text { [64] } \\
\text { - } \\
\text { No sig- } \\
\text { nificant } \\
\text { effect } \\
{[62]}\end{array}$ & - & $\begin{array}{ll}\text { - } & \text { Positive } \\
& {[64]} \\
\text { - } & \text { No signif- } \\
\text { icant ef- } \\
\text { fect [57] }{ }^{\mathrm{c}} \\
(2 \text { stud- } \\
\text { ies })\end{array}$ & - & - \\
\hline $\begin{array}{l}\text { Human (vs } \\
\text { robot) }\end{array}$ & $1(2)$ & $\begin{array}{l}\text { Mixed nega- } \\
\text { tive [62] }\end{array}$ & $\begin{array}{l}\text { Mixed nega- } \\
\text { tive [62] }\end{array}$ & - & $\begin{array}{l}\text { Mixed nega- } \\
\text { tive [62] }\end{array}$ & - & - & - & - \\
\hline $\begin{array}{l}\text { Younger (vs } \\
\text { older) }\end{array}$ & $1(2)$ & $\begin{array}{l}\text { No signifi- } \\
\text { cant effect } \\
{[64]}\end{array}$ & - & $\begin{array}{l}\text { No signifi- } \\
\text { cant effect } \\
{[64]}\end{array}$ & $\begin{array}{l}\text { Mixed posi- } \\
\text { tive [64] }\end{array}$ & - & $\begin{array}{l}\text { No significant } \\
\text { effect [64] }\end{array}$ & - & - \\
\hline Fat (vs slim) & $1(2)$ & Positive [63] & $\begin{array}{l}\text { No signifi- } \\
\text { cant effect } \\
{[63]}\end{array}$ & - & - & - & $\begin{array}{l}\text { No significant } \\
\text { effect [63] }\end{array}$ & - & - \\
\hline $\begin{array}{l}\text { Familiar (vs } \\
\text { unfamiliar) }\end{array}$ & $1(2)$ & $\begin{array}{l}\text { Mixed nega- } \\
\text { tive [64] }\end{array}$ & - & $\begin{array}{l}\text { No signifi- } \\
\text { cant effect } \\
{[64]}\end{array}$ & $\begin{array}{l}\text { Mixed nega- } \\
\text { tive [64] }\end{array}$ & - & $\begin{array}{l}\text { No significant } \\
\text { effect [64] }\end{array}$ & - & - \\
\hline $\begin{array}{l}\text { Medical pro- } \\
\text { fessional at- } \\
\text { tire (vs casu- } \\
\text { al) }\end{array}$ & $1(2)$ & Positive [55] & Positive [55] & - & - & - & Positive [55] & - & - \\
\hline $\begin{array}{l}\text { Medical of- } \\
\text { fice (vs emp- } \\
\text { ty room) }\end{array}$ & $1(2)$ & $\begin{array}{l}\text { Mixed posi- } \\
\text { tive [55] }\end{array}$ & $\begin{array}{l}\text { No signifi- } \\
\text { cant effect } \\
{[55]}\end{array}$ & - & - & - & $\begin{array}{l}\text { No significant } \\
\text { effect [55] }\end{array}$ & - & - \\
\hline $\begin{array}{l}\text { Variability } \\
\text { in camera } \\
\text { angle (vs no } \\
\text { variability) }\end{array}$ & $3(7)$ & $\begin{array}{ll}\text { - } & \text { Mixed } \\
\text { positive } \\
\text { - } 58] \\
\text { - No sig- } \\
\text { nificant } \\
\text { effect } \\
{[58]^{\mathrm{c}}(2} \\
\text { studies })^{2}\end{array}$ & - & - & - & - & - & $\begin{array}{l}\text { No signifi- } \\
\text { cant effect } \\
{[58]^{\mathrm{c}} \text { (3 stud- }} \\
\text { ies) }\end{array}$ & - \\
\hline \multicolumn{10}{|l|}{ Interface design } \\
\hline $\begin{array}{l}\text { Speech input } \\
\text { (vs text or } \\
\text { multiple } \\
\text { choice) }\end{array}$ & $3(7)$ & $\begin{array}{l}\text { No signifi- } \\
\text { cant effect } \\
{[33]}\end{array}$ & $\begin{array}{l}\text { No signifi- } \\
\text { cant effect } \\
{[33]}\end{array}$ & $\begin{array}{l}\text { Mixed nega- } \\
\text { tive [32] }\end{array}$ & - & - & - & Positive [52] & - \\
\hline $\begin{array}{l}\text { Motion initi- } \\
\text { ated (vs user } \\
\text { initiated) }\end{array}$ & $1(2)$ & - & Positive [56] & - & - & $\begin{array}{l}\text { Positive } \\
\text { [56] }\end{array}$ & - & $\begin{array}{l}\text { No signifi- } \\
\text { cant effect } \\
{[56]}\end{array}$ & - \\
\hline
\end{tabular}




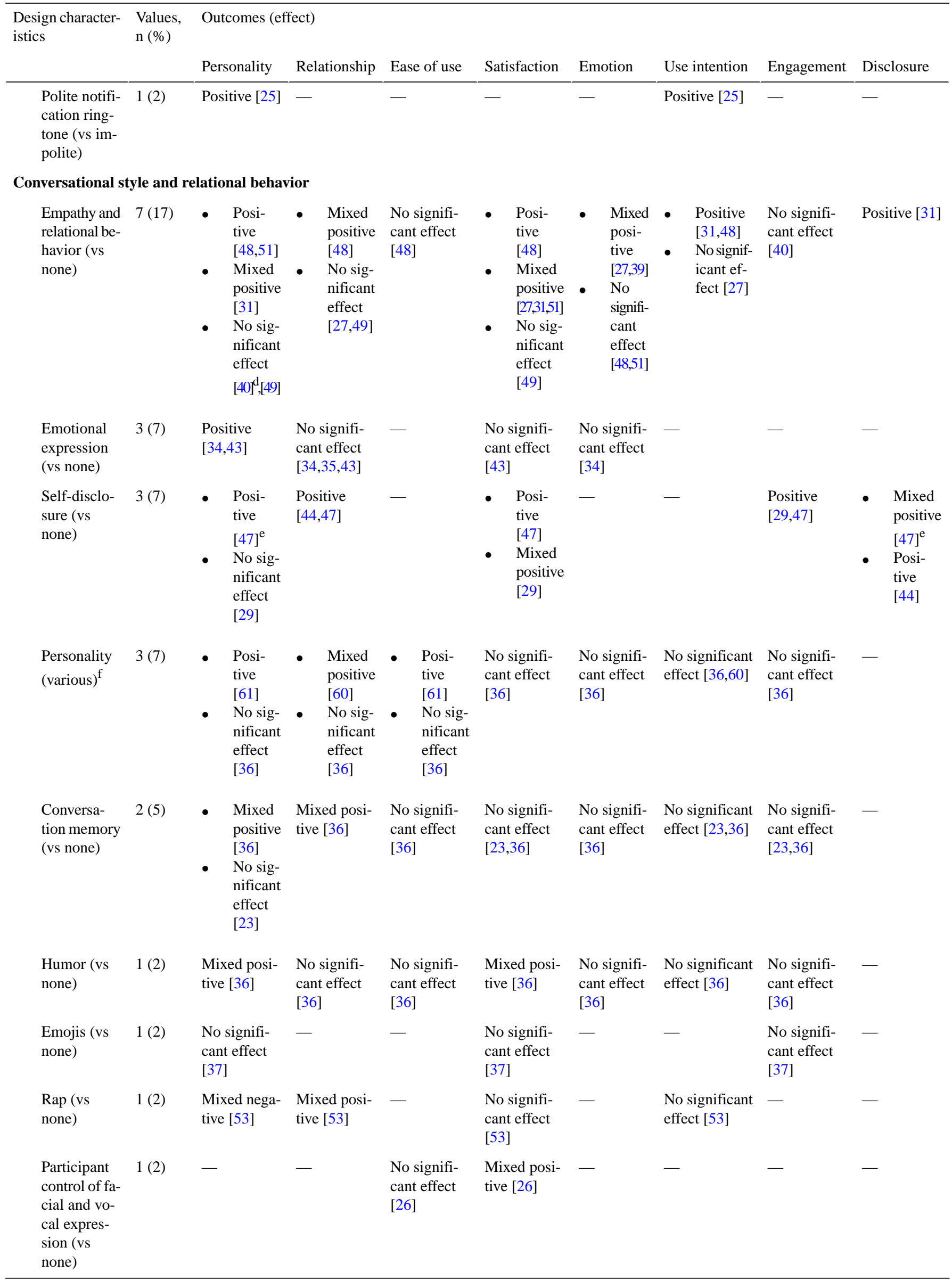




\begin{tabular}{|c|c|c|c|c|c|c|c|c|c|}
\hline \multirow{2}{*}{$\begin{array}{l}\text { Design character- } \\
\text { istics }\end{array}$} & \multirow{2}{*}{$\begin{array}{l}\text { Values, } \\
\mathrm{n}(\%)\end{array}$} & \multicolumn{8}{|c|}{ Outcomes (effect) } \\
\hline & & Personality & Relationship & Ease of use & Satisfaction & Emotion & Use intention & Engagement & Disclosure \\
\hline $\begin{array}{l}\text { Constrained } \\
\text { to positive } \\
\text { user re- } \\
\text { sponse op- } \\
\text { tions (vs } \\
\text { negative re- } \\
\text { sponses al- } \\
\text { lowed) }\end{array}$ & $1(2)$ & $\begin{array}{l}\text { No signifi- } \\
\text { cant effect } \\
{[54]}\end{array}$ & $\begin{array}{l}\text { No signifi- } \\
\text { cant effect } \\
{[54]}\end{array}$ & - & $\begin{array}{l}\text { Mixed nega- } \\
\text { tive [54] }\end{array}$ & - & $\begin{array}{l}\text { No significant } \\
\text { effect [54] }\end{array}$ & - & - \\
\hline \multicolumn{10}{|c|}{ Combined visual and conversational design } \\
\hline $\begin{array}{l}\text { Personifica- } \\
\text { tion (name, } \\
\text { static avatar, } \\
\text { and conversa- } \\
\text { tional lan- } \\
\text { guage vs } \\
\text { none) }\end{array}$ & $1(2)$ & - & - & - & - & - & - & - & $\begin{array}{l}\text { Mixed nega- } \\
\text { tive [59] }\end{array}$ \\
\hline $\begin{array}{l}\text { Variability } \\
\text { in dialog } \\
\text { structure, } \\
\text { language, } \\
\text { and scene } \\
\text { (vs no vari- } \\
\text { ability) }\end{array}$ & $1(2)$ & Positive [29] & - & - & - & - & Positive [29] & Positive [29] & - \\
\hline \multicolumn{10}{|c|}{ Cultural and organizational affiliation } \\
\hline $\begin{array}{l}\text { Culturally } \\
\text { tailored argu- } \\
\text { mentation } \\
\text { (vs not) }\end{array}$ & $2(5)$ & $\begin{array}{l}\text { No signifi- } \\
\text { cant effect } \\
{[65]}\end{array}$ & - & - & Positive [50] & - & - & - & - \\
\hline $\begin{array}{l}\text { Culturally } \\
\text { tailored ap- } \\
\text { pearance (vs } \\
\text { not) }\end{array}$ & $3(7)$ & $\begin{array}{l}\text { No signifi- } \\
\text { cant effect } \\
{[65,67]}\end{array}$ & $\begin{array}{l}\text { Negative } \\
{[67]}\end{array}$ & $\begin{array}{l}\text { No signifi- } \\
\text { cant effect } \\
{[67]}\end{array}$ & $\begin{array}{l}\text { No signifi- } \\
\text { cant effect } \\
{[50,67]}\end{array}$ & - & $\begin{array}{l}\text { No significant } \\
\text { effect [67] }\end{array}$ & - & - \\
\hline $\begin{array}{l}\text { Culturally } \\
\text { tailored argu- } \\
\text { mentation } \\
\text { and scene } \\
\text { combined } \\
\text { (vs not) }\end{array}$ & $1(2)$ & $\begin{array}{l}\text { No signifi- } \\
\text { cant effect } \\
{[68]}\end{array}$ & $\begin{array}{l}\text { No signifi- } \\
\text { cant effect } \\
{[68]}\end{array}$ & $\begin{array}{l}\text { No signifi- } \\
\text { cant effect } \\
{[68]}\end{array}$ & $\begin{array}{l}\text { No signifi- } \\
\text { cant effect } \\
{[68]}\end{array}$ & - & $\begin{array}{l}\text { No significant } \\
\text { effect [68] }\end{array}$ & - & - \\
\hline $\begin{array}{l}\text { Patient assis- } \\
\text { tant (vs re- } \\
\text { searcher or } \\
\text { government } \\
\text { employee) }\end{array}$ & $1(2)$ & $\begin{array}{l}\text { Mixed posi- } \\
\text { tive [66] }\end{array}$ & - & - & $\begin{array}{l}\text { Mixed posi- } \\
\text { tive [66] }\end{array}$ & - & Positive [66] & - & - \\
\hline
\end{tabular}

${ }^{a}$ Results indicated for nonempathetic avatar only (empathetic avatar had additional dialog to the no avatar condition).

${ }^{\mathrm{b}}$ No study examined the combination of design characteristic and outcome.

${ }^{\mathrm{c}}$ Multiple studies were reported in the article with similar results.

${ }^{\mathrm{d}}$ Similar results were additionally reported at a different time point in the study [39].

${ }^{\text {e }}$ Similar results were additionally reported at a different time point in the study [46].

${ }^{\mathrm{f}}$ Indicates any effects of personality (no consistent comparator).

Table 3 summarizes research findings grouped according to the design characteristic examined and the categories of outcomes measured. Where identical outcomes of a study were reported in multiple articles, the primary reference listed in Multimedia Appendix 2 was used. Additional references were used for outcomes that were not reported in the primary study. In total, 4 studies did not present a statistical analysis comparing the relevant experimental conditions; therefore, these studies are not included in Table 3.

The following paragraphs highlight key results from the studies presented in Table 3 and include a narrative synthesis of studies that were not presented in Table 3. 


\section{Visual Design}

Approximately 7\% (3/41) of studies examined whether user experience differed using a virtual assistant with an animated avatar compared with using a text- or speech-only virtual assistant with no visual representation [48,51,62]. Findings were generally nonsignificant $[48,51,62]$, with some mixed negative effects of using an animated avatar [48,62]. An additional study not included in Table 3 concluded that virtual assistants with an animated avatar were preferred over voice-only assistants; however, the analyses included both real and virtual assistants [45].

Approximately $22 \%$ (9/41) of studies examined the appearance of the animated avatar, and $10 \%(4 / 41)$ of studies examined whether user experience differed using a virtual assistant with a more realistic human avatar compared with a more cartoon human avatar [57,62,64]. Although some positive and mixed positive effects of using a more realistic avatar were found [64], more effects were nonsignificant [57,62,64], and 1 was negative [57]. The species of the avatar was examined by $2 \%(1 / 41)$ of studies, which found mixed negative effects of using a human avatar compared with using a robot avatar [62]. Age was examined by $2 \%$ (1/41) of studies, which found mixed positive effects of using an avatar with a younger appearance compared with using one with an older appearance on satisfaction but no significant effects on other outcomes [64]. Body shape was examined by $2 \%(1 / 41)$ of studies, which found a positive effect of a fat avatar compared with a slim avatar on personality traits but nonsignificant effects on other outcomes [63]. The familiarity of the avatar was examined by $2 \%(1 / 41)$ of studies, which found mixed negative and nonsignificant effects of using an avatar that looked like a health coach that participants met at the beginning of the session compared with using an unfamiliar avatar [64]. The avatar's attire was examined by $2 \%$ (1/41) of studies, which found consistently positive effects of medical professional attire compared with casual attire [55].

The background scene behind the avatar was examined by $2 \%$ (1/41) of studies, which found mixed positive effects of representing a medical office compared with representing an empty room on personality but no significant effects on other measured outcomes [55]. Approximately 7\% (3/41) of studies (all reported in 1 paper) examined whether variability in the camera position, distance, and focus was associated with user experience and found mostly nonsignificant effects [58].

\section{Interface Design}

Approximately $7 \%$ (3/41) of studies examined the effects of input modality - whether the user communicates using speech, text, or multiple choice- on user experience and found a combination of positive, mixed negative, and nonsignificant effects of speech input compared with other modalities $[32,33,52]$. A menu-based virtual assistant was examined by 1 further study not included in Table 3, and it concluded that there were no differences in usability between speech and phone key press user input [42].

How the conversation between the virtual assistant and user was initiated was examined by $2 \%(1 / 41)$ of studies, which found positive and nonsignificant effects of automated motion initiation compared with user initiation [56]. The type of ringtone used to initiate a conversation with the user was examined by $2 \%(1 / 41)$ of studies, which found positive effects of more polite tones compared with less polite tones [25].

\section{Conversational Style and Relational Behavior}

Approximately 17\% (7/41) of studies examined empathy and relational behavior-empathetic verbal feedback and nonverbal behavior such as facial expressions and gestures $[27,31,39,40,48,49,51]$. Although some effects were nonsignificant $[27,40,48,49,51]$, more effects were positive or mixed positive, with $71 \%(5 / 7)$ of studies showing at least some positive effect $[27,31,39,48,51]$. Approximately $7 \%$ (3/41) of studies examined emotional expression-the use of facial expression and voice to express emotion-and found some mixed positive effects $[34,43]$ but more nonsignificant effects $[34,35,43]$. Approximately 7\% (3/41) of studies examined self-disclosure-whether the virtual assistant tells the user information about themselves-and found mostly positive effects [29,44,47]. Approximately 7\% (3/41) of studies examined personality $[36,60,61]$. Although some positive and mixed positive effects were found $[60,61]$, most effects were nonsignificant $[36,60]$.

Approximately 5\% (2/41) of studies examined conversation memory - whether the virtual assistant remembered information from earlier conversation-and found some mixed positive effects [36] but mostly nonsignificant effects [23,36]. An additional study not included in Table 3 compared users' first interactions when the virtual assistant did not recall their previous session and when the virtual assistant did recall their previous session [38]. The authors concluded that users were more positive when the virtual assistant recalled their session; however, the conversations were less personal.

Humor was examined by $2 \%(1 / 41)$ of studies, which found mostly nonsignificant effects of including humor compared with not including humor [36]. Using emojis was examined by $2 \%(1 / 41)$ of studies, which found no significant effects of using emojis compared with not using emojis [37]. Rap was examined by $2 \%(1 / 41)$ of studies, which found a combination of mixed positive, mixed negative, and nonsignificant effects of including rap compared with not including rap [53]. Allowing participants to control the virtual assistant's facial and vocal expression was examined by $2 \%$ (1/41) of studies, which found nonsignificant and mixed positive effects compared with not allowing such control [26]. Approximately 2\% (1/41) of studies examined constraining users to respond only positively to questions about their confidence and motivation compared with also presenting negative multiple-choice response options [54]. It found a combination of mixed negative and neutral effects of constraining users to positive responses. A further study not included in Table 3 examined whether user evaluations were more positive for a virtual assistant that changed behavior based on the user's eye contact compared with a virtual assistant that always appeared attentive or always bored or that changed behavior randomly [41]. The authors concluded that changing based on the user's eye contact seemed more normal than changing behavior randomly but did not confirm the hypothesis 
that changing behavior is more normal than unchanging behavior.

\section{Combined Visual and Conversational Design}

Personification - the use of a name, static avatar, and conversational language-was examined by $2 \%(1 / 41)$ of studies, which found negative effects of personification on users' disclosure [59]. Variability in dialog structure (the order of the conversation and the utterances used) and background scene was examined by $2 \%(1 / 41)$ of studies, which found consistently positive effects of variability compared with no variability [29].

\section{Organizational and Cultural Affiliation}

Approximately $10 \%$ (4/41) of studies examined cultural tailoring - matching the culture of the virtual assistant to that of the user [50,65,67,68]. Approximately 5\% (2/41) of studies examined cultural tailoring of the virtual assistant's argumentation (eg, discussed culturally relevant topics) [50,65], and $50 \%(1 / 2)$ of those found a positive effect [50]. Approximately 7\% (3/41) of studies examined cultural tailoring of the virtual assistant's appearance and the household setting and found predominantly nonsignificant effects [50,65,67]. Culturally tailored background scene and argumentation combined were examined by $2 \%$ (1/41) of studies, which found no significant effects [68]. The organizational affiliation of the virtual assistant - who the virtual assistant claimed to be and the context provided in the background scene-was examined by $2 \%(1 / 41)$ of studies, which found positive effects of the virtual assistant being a patient assistant compared with the virtual assistant being either a member of the medical team conducting the research or a government employee [66].

\section{Discussion}

\section{Principal Findings}

This study aimed to provide an overview of experimental research examining how design characteristics of virtual health assistants affect user experience. This is a growing area of scientific endeavor with studies, taken together, examining highly diverse health domains, design characteristics, and outcomes. The most common health domains were physical activity and mental health, with relatively few virtual assistants related to specific health conditions. Approximately half of the studies were categorized as examining the design of conversational style and relational behavior, with the most common design characteristic researched being empathy and relational behavior. The most commonly measured outcomes were in the categories of personality traits, satisfaction, relationship, and use intention.

This study also aimed to summarize the research findings of experimental research examining how design characteristics of virtual health assistants affect user experience. Generally, research has been piecemeal, with few design characteristics having a sufficient body of evidence to draw conclusions about their effects on user experience. The 2 design characteristics that defy this are virtual assistants' empathy and relational behavior and self-disclosure, which have been the focus of a good number of studies. Research suggests that all 3 (ie, empathy, relational behavior, and self-disclosure) are related to more positive user experience. Other design characteristics with emerging levels of evidence are having a more realistic human representation for an avatar and having medical attire for the avatar, both of which may potentially be related to more positive user experience. Finally, evidence to date suggests that using an animated avatar (compared with no avatar) and cultural tailoring may not affect user experience; however, more research is needed to explore these findings.

One of the clearest findings of this study was that the use of empathy and relational behavior in virtual health assistants appears to have positive effects on user experience. Empathy may help to build trust and rapport with the virtual assistant. The finding that empathy was associated with user satisfaction is in line with research indicating a positive association between empathy in real health care providers and patient satisfaction $[69,70]$. Results were not consistently positive; however, this may be related to differences between the virtual assistants. For example, for the outcome category personality traits, of the 5 studies examining empathy and relational behavior, $3(60 \%)$ studies showing positive effects used animated avatars, including nonverbal relational behaviors [31,48,51]. In contrast, $40 \%(2 / 5)$ of studies showing no effects were text-only assistants [40,49]. It may be that users do not expect text-only assistants to show empathy; therefore, the presence or absence of empathy has no impact on the ratings of the virtual assistant. Alternatively, the effects of empathy may be diminished when nonverbal relational behaviors such as expression and gestures are not present.

Research suggests that virtual health assistants that use self-disclosure (ie, provide information about themselves) elicit a more positive user experience. Results were similar whether the autobiographical information was framed as being about the virtual assistant's experience as a computer agent [44] or included human experiences that could not actually be true $[29,47]$. Self-disclosure is important for the formation of relationships [71], although research suggests that self-disclosure by a real counselor can have either positive or detrimental effects on a client's perceptions of the counselor [72]. The finding that users respond positively to the autobiographical stories of a virtual health assistant supports the computers are social actors paradigm, where users display social responses to computers, although they know they are not human $[73,74]$.

Research examining the realism of the animated avatar showed some positive effects; however, more were nonsignificant. The uncanny valley theory suggests that robots that appear almost but not quite human may elicit a negative emotional response and be less likable than those that are clearly nonhuman [75]. However, in this review, the study that used a photo-realistic representation in the realistic experimental condition [64] showed positive effects. More research is needed to examine how the realism of the avatar affects the user experience of virtual health assistants.

Results from 1 study suggest that dressing the avatar in medical attire results in a more positive user experience [55]. Although more research is needed to confirm this finding, this was a large study $(n=308)$ with consistent results across all outcomes measures. Interestingly, the background setting for the avatar 
(medical office or empty room) had a mixed positive effect on only 1 out of 3 outcomes categories [55].

Research suggests that including an animated avatar has no effect or, in some cases, a negative effect on user experience. However, upon closer inspection, this may be because of the nature of the avatars used in the research and may also be affected by interactions between the animation and other virtual assistant characteristics. For example, Lisetti et al [48] showed that an animated avatar with a neutral facial expression and no empathetic dialog led to poorer user experience than a text-only virtual assistant, whereas an expressive and empathetic virtual assistant led to a better user experience than the text-only virtual assistant. Nguyen and Masthoff [51] reported similar findings; a nonempathetic animated virtual assistant and a nonempathetic text-only virtual assistant led to a similar user experience; however, an empathetic animated virtual assistant led to better user experience than an empathetic text-only virtual assistant. Taken together, it appears that users may expect a virtual assistant with a human-like representation to have empathy and human-like relational behaviors and have a poorer user experience when this expectation is not met

Overall, the research did not show cultural tailoring to improve the user experience of virtual health assistants. Notably, although $75 \%(3 / 4)$ of studies included participants who were born overseas (in China [68], India [50], or a Spanish-speaking Latin-American country [65]), participants in all the studies lived in the United States. This may suggest that cultural tailoring is not required for different cultures living in the United States who have had exposure to Anglo-American culture, although more research could confirm this finding. Additional research is also needed to determine whether cultural tailoring affects user experience in other cultural contexts.

\section{Strengths and Limitations}

This scoping review is the most rigorous attempt at synthesizing the literature regarding the effects of design characteristics on the user experience of virtual health assistants. It followed the PRISMA-ScR guidelines for scoping reviews and searched a large number of databases. It examined a broad range of design characteristics using the highest level of evidence-experimental research using only interactive virtual health assistants where participants were able to input into the system. However, we acknowledge that the use of specific search terms to capture virtual assistants and design characteristics could have omitted some results. It is also possible that other literary sources may have been available in other databases. In addition, qualitative data were excluded. This enabled a structured approach to synthesizing the data based on statistical significance but may have omitted some important views on user experience.

Although the breadth of the review is a major strength, the heterogeneity of the included studies makes it difficult to synthesize and interpret the results. There was considerable heterogeneity in the purpose of the virtual assistants studied. Optimal design techniques may differ among different health domains. For example, although no overall effect of using emojis was found, the difference in ratings of confidence between using text-only and text with emojis depended on whether the virtual assistant was discussing physical or mental well-being [37]. In addition, some health conditions were not represented in the studies, for example, neurocognitive impairments such as dementia. There was also significant heterogeneity in the outcomes measured. The most commonly measured outcomes were in the categories of personality, satisfaction, relationship, and use intention. Few studies examined the ease of use, engagement, or disclosure. Although interface design may play a key role in determining the ease of use, other design characteristics such as the visual appearance of an avatar may not be expected to affect the ease of use. More research examining how users interact with the virtual assistant (engagement and disclosure), particularly using objective measures, may complement subjective ratings of the virtual assistant and interaction.

An additional limitation of the literature is that some studies combined a set of similar characteristics into 1 condition, making it difficult to ascertain which characteristic might be responsible for the effects on user experience. For example, research on empathy and relational behavior frequently included verbal empathy with nonverbal relational behaviors. In addition, in most studies, participants evaluated the virtual assistant after interacting during a single session. Programs that aim to promote health behavior change or provide support for a health condition are often designed for ongoing use. Additional research should examine how design characteristics affect user experience over time. Most virtual assistants had animated avatars and speech output; however, over half constrained user input to selecting from predefined response options. Constraining user input requires simpler programming and removes the risk of errors occurring when the virtual assistant misinterprets the user's input or cannot formulate a response to a query that is outside the bounds of its programmed knowledge [76]. Natural language processing enables users to communicate using unconstrained text or speech and enables more natural user-directed communication. Virtual assistants using natural language processing have been commonly used in health care [77] and, with rapid advancements in artificial intelligence, are likely to become increasingly sophisticated. More research should examine the design and user experience of these types of virtual health assistants

\section{Recommendations}

Research demonstrates that design characteristics affect the user experience of virtual health assistants; therefore, researchers and software developers should carefully consider the look and feel of a virtual health assistant during development and testing. On the basis of the results of this scoping review, the following recommendations for designing virtual health assistants and advancing the field of research may be useful for health researchers and software developers:

1. Design virtual health assistants to express verbal empathy, for example, understanding of the user's feelings

2. Design virtual health assistants to disclose personal information about themselves to the user, for example, information about their past and personal preferences

3. Consider designing a human avatar to be more realistic with medical professional attire 
4. If designing an animated virtual health assistant, it should display nonverbal relational behaviors, for example, emotional facial expressions, gestures, and mutual gaze

5. If empathy and relational behaviors are unable to be incorporated, consider that an animated avatar may not be beneficial or cost-effective

6. Engage in formative research with the target audience and adopt a user-centered design approach to ensure that the software meets the needs and preferences of the user

7. Conduct further systematic research to replicate and extend previous findings, particularly with longitudinal research designs with repeated user interactions, objective engagement outcomes, and virtual assistants with natural language processing capabilities

\section{Conclusions}

Virtual health assistants can provide health information and support on demand and may be applied in the future to a wide variety of purposes such as providing public health information, health education, supporting patients with chronic health conditions, and assisting with healthy lifestyle behavior change. This scoping review examined experimental research assessing how design characteristics of virtual health assistants affect user experience. This is a rapidly growing field of research but is difficult to synthesize and interpret because of the heterogeneity of studies. Nonetheless, certain design characteristics have emerged as important for improving user experience. Preliminary recommendations suggest that programming virtual health assistants to show empathy, display nonverbal relational behaviors, and disclose personal information about themselves may result in a more positive user experience. The decision to include an animated avatar should consider whether the avatar can display empathy and nonverbal relational behaviors. Future research is required to improve our understanding of the relationship between design characteristics and user experience of virtual health assistants, particularly with longitudinal research designs with repeated user interactions.

\section{Acknowledgments}

CAM is supported by a Medical Research Future Fund Emerging Leader Grant (GNT1193862). HTB is supported by an Australian Government Research Training Program Scholarship. The funders had no role in the study design, data collection and analysis, decision to publish, or preparation of the manuscript.

\section{Authors' Contributions}

RGC and CAM conceived and designed the study. RGC conducted database searches. RGC and BB conducted screening, and RGC, BB, TF, CN, HTB, and RV conducted data extraction. RGC analyzed the data and wrote the manuscript. All authors revised the manuscript and reviewed and approved the final version.

\section{Conflicts of Interest}

None declared.

\section{Multimedia Appendix 1}

Data charting form.

[XLSX File (Microsoft Excel File), 10 KB-Multimedia Appendix 1]

\section{Multimedia Appendix 2}

Characteristics of studies included in the scoping review.

[DOCX File , 56 KB-Multimedia Appendix 2]

\section{Multimedia Appendix 3}

Outcome categories.

\section{[DOCX File, 17 KB-Multimedia Appendix 3]}

\section{References}

1. Horgan D, Romao M, Morré SA, Kalra D. Artificial intelligence: power for civilisation - and for better healthcare. Public Health Genomics 2019;22(5-6):145-161 [FREE Full text] [doi: 10.1159/000504785] [Medline: $\underline{31838476}$ ]

2. Fitzpatrick KK, Darcy A, Vierhile M. Delivering cognitive behavior therapy to young adults with symptoms of depression and anxiety using a fully automated conversational agent (Woebot): a randomized controlled trial. JMIR Ment Health 2017 Jun 06;4(2):e19 [FREE Full text] [doi: 10.2196/mental.7785] [Medline: 28588005]

3. Maher CA, Davis CR, Curtis RG, Short CE, Murphy KJ. A physical activity and diet program delivered by artificially intelligent virtual health coach: proof-of-concept study. JMIR Mhealth Uhealth 2020 Jul 10;8(7):e17558 [FREE Full text] [doi: 10.2196/17558] [Medline: 32673246]

4. Cleres D, Rassouli F, Brutsche M, Kowatsch T, Barata F. Lena: a voice-based conversational agent for remote patient monitoring in chronic obstructive pulmonary disease.; April 13-17 2021; College Station, USA. In: Proceedings of the 26th 
ACM Conference on Intelligent User Interfaces. 2021 Presented at: 26th ACM Conference on Intelligent User Interfaces; Apr 13-17, 2021; College Station, USA. [doi: 10.3929/ethz-b-000472586]

5. Ergonomics of human-system interaction - Part 11: Usability: Definitions and concepts. ISO. 2018. URL: https://www. iso.org/obp/ui/\#iso:std:iso:9241:-11:ed-2:v1:en [accessed 2021-11-29]

6. Thüring M, Mahlke S. Usability, aesthetics and emotions in human-technology interaction. Int J Psychol 2007 Aug;42(4):253-264. [doi: 10.1080/00207590701396674]

7. Bhandari U, Chang K, Neben T. Understanding the impact of perceived visual aesthetics on user evaluations: an emotional perspective. Inf Manag 2019 Jan;56(1):85-93. [doi: 10.1016/j.im.2018.07.003]

8. Pearl C. Designing Voice User Interfaces: Principles of Conversational Eperiences. Beijing, China: O'Reilly Media Incorporated; 2016.

9. Lister K, Coughlan T, Iniesto F, Freear N, Devine P. Accessible conversational user interfaces: considerations for design. In: Proceedings of the 17th International Web for All Conference. 2020 Presented at: Proceedings of the 17th International Web for All Conference; Apr 20-21 2020; Taipei, Taiwan. [doi: 10.1145/3371300.3383343]

10. Wellnhammer N, Dolata M, Steigler S, Schwabe G. Studying with the help of digital tutors: design aspects of conversational agents that influence the learning process. In: Proceedings of the 53rd Hawaii International Conference on System Sciences. 2020 Presented at: 53rd Hawaii International Conference on System Sciences; Jan 2020; Hawaii, USA. [doi:

10.24251/hicss.2020.019]

11. Strohmann T, Siemon D, Robra-Bissantz S. Designing virtual in-vehicle assistants: design guidelines for creating a convincing user experience. AIS Trans Human Comput Interac 2019;11(2):54-78. [doi: 10.17705/1thci.00113]

12. Følstad A, Skjuve M, Brandtzaeg P. Different chatbots for different purposes: towards a typology of chatbots to understand interaction design. In: Internet Science. Cham: Springer; 2019.

13. Janssen A, Passlick J, Rodríguez Cardona D, Breitner MH. Virtual assistance in any context. Bus Inf Syst Eng 2020 Apr 06;62(3):211-225. [doi: 10.1007/s12599-020-00644-1]

14. Holmes S, Moorhead A, Bond R, Zheng H, Coates V, Mctear M. Usability testing of a healthcare chatbot: can we use conventional methods to assess conversational user interfaces? In: Proceedings of the 31 st European Conference on Cognitive Ergonomics. 2019 Presented at: 31st European Conference on Cognitive Ergonomics; Sept 10-13, 2019; Belfast, UK. [doi: 10.1145/3335082.3335094]

15. ter Stal S, Kramer LL, Tabak M, op den Akker H, Hermens H. Design features of embodied conversational agents in eHealth: a literature review. Int J Human Comput Stud 2020 Jun;138:102409. [doi: 10.1016/j.ijhcs.2020.102409]

16. Munn Z, Peters MD, Stern C, Tufanaru C, McArthur A, Aromataris E. Systematic review or scoping review? Guidance for authors when choosing between a systematic or scoping review approach. BMC Med Res Methodol 2018 Nov 19;18(1):143 [FREE Full text] [doi: 10.1186/s12874-018-0611-x] [Medline: 30453902]

17. Tricco AC, Lillie E, Zarin W, O'Brien KK, Colquhoun H, Levac D, et al. PRISMA Extension for Scoping Reviews (PRISMA-ScR): checklist and explanation. Ann Intern Med 2018 Oct 02;169(7):467-473 [FREE Full text] [doi: 10.7326/M18-0850] [Medline: 30178033]

18. Richardson WS, Wilson MC, Nishikawa J, Hayward RS. The well-built clinical question: a key to evidence-based decisions. ACP J Club 1995;123(3):A12-A13. [Medline: 7582737]

19. Dahlbäck N, Jönsson A, Ahrenberg L. Wizard of Oz studies — why and how. Knowl Based Syst 1993 Dec;6(4):258-266. [doi: $10.1016 / 0950-7051(93) 90017-n$ ]

20. Endnote Team. EndNote. EndNote X9 ed. Philadelphia, PA: Clarivate; 2013.

21. Amini R, Lisetti C, Yasavur U. Emotionally responsive virtual counselor for behavior-change health interventions. In: Advancing the Impact of Design Science: Moving from Theory to Practice. Cham: Springer International Publishing; 2014.

22. Amini R, Lisetti C, Yasavur U, Rishe N. On-demand virtual health counselor for delivering behavior-change health interventions. In: Proceedings of the IEEE International Conference on Healthcare Informatics. 2013 Presented at: IEEE International Conference on Healthcare Informatics; Sep 9-11, 2013; Philadelphia, PA, USA. [doi: 10.1109/ichi.2013.13]

23. Bellur S, Sundar SS. Talking health with a machine: how does message interactivity affect attitudes and cognitions? Hum Commun Res 2016 Sep 15;43(1):25-53. [doi: 10.1111/hcre.12094]

24. Bickmore T, Gruber A, Picard R. Establishing the computer-patient working alliance in automated health behavior change interventions. Patient Educ Couns 2005 Oct;59(1):21-30. [doi: 10.1016/j.pec.2004.09.008] [Medline: 16198215]

25. Bickmore T, Mauer D, Crespo F, Brown T. Persuasion, task interruption and health regimen adherence. In: Proceedings of the Persuasive Technology, Second International Conference on Persuasive Technology, PERSUASIVE 2007. 2007 Presented at: Persuasive Technology, Second International Conference on Persuasive Technology, PERSUASIVE 2007; Apr 26-27, 2007; Palo Alto, CA, USA. [doi: 10.1007/978-3-540-77006-0 1]

26. Bickmore T, Ring L. Making it personal: end-user authoring of health narratives delivered by virtual agents. In: Proceedings of the Intelligent Virtual Agents, 10th International Conference, IVA 2010. 2010 Presented at: Intelligent Virtual Agents, 10th International Conference, IVA 2010; Sep 20-22, 2010; Philadelphia, PA, USA. [doi: 10.1007/978-3-642-15892-6 43]

27. Bickmore T, Schulman D. Practical approaches to comforting users with relational agents. In: Proceedings of the CHI Conference on Human Factors in Computing. 2007 Presented at: CHI07: CHI Conference on Human Factors in Computing; Apr 28-May 3, 2007; San Jose, CA, USA. [doi: 10.1145/1240866.1240996] 
28. Bickmore T, Schulman D, Yin L. Engagement vs. deceit: virtual humans with human autobiographies. In: Proceedings of the Intelligent Virtual Agents, 9th International Conference, IVA 2009. 2009 Presented at: Intelligent Virtual Agents, 9th International Conference, IVA 2009; Sep 14-16, 2009; Amsterdam, The Netherlands. [doi: 10.1007/978-3-642-04380-2 4]

29. Bickmore T, Schulman D, Yin L. Maintaining engagement in long-term interventions with relational agents. Appl Artif Intell 2010 Jul 01;24(6):648-666 [FREE Full text] [doi: 10.1080/08839514.2010.492259] [Medline: 21318052]

30. Bickmore T, Picard R. Towards caring machines. In: Proceedings of the CHI 2004 Conference on Human Factors in Computing. 2004 Presented at: CHI 2004 Conference on Human Factors in Computing; Apr 24-29, 2004; Vienna, Austria. [doi: 10.1145/985921.986097]

31. Bickmore TW, Picard RW. Establishing and maintaining long-term human-computer relationships. ACM Trans Comput Hum Interact 2005 Jun 01;12(2):293-327. [doi: 10.1145/1067860.1067867]

32. Chen J, Lyell D, Laranjo L, Magrabi F. Effect of speech recognition on problem solving and recall in consumer digital health tasks: controlled laboratory experiment. J Med Internet Res 2020 Jun 01;22(6):e14827 [FREE Full text] [doi: 10.2196/14827] [Medline: 32442129]

33. Cho E. Hey Google, can i ask you something in private? In: CHI '19: Proceedings of the 2019 CHI Conference on Human Factors in Computing Systems. 2019 Presented at: CHI '19: CHI Conference on Human Factors in Computing Systems; May 4 - 9, 2019; Glasgow Scotland UK. [doi: 10.1145/3290605.3300488]

34. Creed C, Beale R. User interactions with an affective nutritional coach. Interact Comput 2012 Sep;24(5):339-350. [doi: 10.1016/j.intcom.2012.05.004]

35. Creed C, Beale R, Cowan B. The impact of an embodied agent's emotional expressions over multiple interactions. Interact Comput 2014 Jan 29;27(2):172-188. [doi: 10.1093/iwc/iwt064]

36. De Boni M, Richardson A, Hurling R. Humour, relationship maintenance and personality matching in automated dialogue: a controlled study. Interact Comput 2008 May;20(3):342-353. [doi: 10.1016/j.intcom.2007.11.002]

37. Fadhil A, Schiavo G, Wang Y, Yilma B. The effect of emojis when interacting with conversational interface assisted health coaching system. In: Proceedings of the 12th EAI International Conference on Pervasive Computing Technologies for Healthcare. 2018 Presented at: Pervasive Health '18: 12th EAI International Conference on Pervasive Computing Technologies for Healthcare; May 21 - 24, 2018; New York NY USA. [doi: 10.1145/3240925.3240965]

38. Ferland L, Koutstaal W. How's your day look? The (un)expected sociolinguistic effects of user modeling in a conversational agent. In: Proceedings of the 2020 CHI Conference on Human Factors in Computing Systems. 2020 Presented at: CHI '20: CHI Conference on Human Factors in Computing Systems; Apr 25-30, 2020; Honolulu, HI, USA. [doi: $10.1145 / 3334480.3375227]$

39. Ghandeharioun A, McDuff D, Czerwinski M, Rowan K. Towards understanding emotional intelligence for behavior change chatbots. In: Proceedings of the 8th International Conference on Affective Computing and Intelligent Interaction. 2019 Presented at: 8th International Conference on Affective Computing and Intelligent Interaction; Sep 3-6, 2019; Cambridge, UK. [doi: 10.1109/acii.2019.8925433]

40. Ghandeharioun A, McDuff D, Czerwinski M, Rowan K. EMMA: an emotion-aware wellbeing chatbot. In: Proceedings of the 8th International Conference on Affective Computing and Intelligent Interaction. 2019 Presented at: 8th International Conference on Affective Computing and Intelligent Interaction; Sep 3-6, 2019; Cambridge, UK. [doi: 10.1109/acii.2019.8925455]

41. Grillon H, Thalmann D. Eye contact as trigger for modification of virtual character behavior. In: Proceedings of the Virtual Rehabilitation. 2008 Presented at: Virtual Rehabilitation; Aug 25-27, 2008; Vancouver, BC, Canada. [doi: 10.1109/icvr.2008.4625161]

42. Grover A, Plauché M, Barnard E, Kuun C. HIV health information access using spoken dialogue systems: touchtone vs. speech. In: Proceedings of the International Conference on Information and Communication Technologies and Development. 2009 Presented at: International Conference on Information and Communication Technologies and Development; Apr 17-19, 2009; Doha, Qatar. [doi: 10.1109/ictd.2009.5426716]

43. Guadagno RE, Swinth KR, Blascovich J. Social evaluations of embodied agents and avatars. Comput Human Behav 2011 Nov;27(6):2380-2385. [doi: 10.1016/j.chb.2011.07.017]

44. Kang S, Gratch J. People like virtual counselors that highly-disclose about themselves. Stud Health Technol Inform 2011;167:143-148. [Medline: 21685657]

45. Kim K, Norouzi N, Losekamp T, Bruder G, Anderson M, Welch G. Effects of patient care assistant embodiment and computer mediation on user experience. In: Proceedings of the 2019 IEEE International Conference on Artificial Intelligence and Virtual Reality (AIVR). 2019 Presented at: 2019 IEEE International Conference on Artificial Intelligence and Virtual Reality (AIVR); Dec 9-11, 2019; San Diego, CA. [doi: 10.1109/aivr46125.2019.00013]

46. Lee Y, Yamashita N, Huang Y. Designing a chatbot as a mediator for promoting deep self-disclosure to a real mental health professional. Proc ACM Human Comput Interact 2020 May 28;4(1):1-27. [doi: 10.1145/3392836]

47. Lee Y, Yamashita N, Huang Y, Fu W. "I Hear You, I Feel You": encouraging deep self-disclosure through a chatbot. In: Proceedings of the 2020 CHI Conference on Human Factors in Computing Systems. 2020 Presented at: 2020 CHI Conference on Human Factors in Computing Systems; Apr 25-30, 2020; Honolulu, HI, USA. [doi: 10.1145/3313831.3376175] 
48. Lisetti C, Amini R, Yasavur U, Rishe N. I can help you change! An empathic virtual agent delivers behavior change health interventions. ACM Trans Manage Inf Syst 2013 Dec 01;4(4):1-28. [doi: 10.1145/2544103]

49. Liu B, Sundar SS. Should machines express sympathy and empathy? Experiments with a health advice chatbot. Cyberpsychol Behav Soc Netw 2018 Oct;21(10):625-636. [doi: 10.1089/cyber.2018.0110] [Medline: 30334655]

50. Murali P, Shamekhi A, Parmar D, Bickmore T. Argumentation is more important than appearance for designing culturally tailored virtual agents. In: Proceedings of the 19th International Conference on Autonomous Agents and MultiAgent Systems. 2020 Presented at: 19th International Conference on Autonomous Agents and MultiAgent Systems; May 9 - 13, 2020; Auckland, New Zealand. [doi: 10.1145/860575.860717]

51. Nguyen H, Masthoff J. Designing empathic computers: the effect of multimodal empathic feedback using animated agent. In: Proceedings of the 4th International Conference on Persuasive Technology. 2009 Presented at: 4th International Conference on Persuasive Technology; Apr 26-29, 2009; Claremont, CA, USA. [doi: 10.1145/1541948.1541958]

52. Novielli N, de Rosis F, Mazzotta I. User attitude towards an embodied conversational agent: effects of the interaction mode. J Pragmatics 2010 Sep;42(9):2385-2397. [doi: 10.1016/j.pragma.2009.12.016]

53. Olafsson S, Kimani E, Asadi R, Bickmore T. That's a rap. In: Intelligent Virtual Agents. Cham: Springer; 2017.

54. Olafsson S, O'Leary T, Bickmore T. Coerced change-talk with conversational agents promotes confidence in behavior change. In: Proceedings of the 13th International Conference on Pervasive Computing Technologies for Healthcare. 2019 Presented at: 13th International Conference on Pervasive Computing Technologies for Healthcare; May 20-23, 2019; Trento, Italy. [doi: 10.1145/3329189.3329202]

55. Parmar D, Olafsson S, Utami D, Bickmore T. Looking the part: the effect of attire and setting on perceptions of a virtual health counselor. In: Proceedings of the 18th ACM International Conference on Intelligent Virtual Agents. 2018 Presented at: 18th ACM International Conference on Intelligent Virtual Agents; Nov 5-8, 2018; Sydney, Australia. [doi: $10.1145 / 3267851.3267915]$

56. Ring L, Shi L, Totzke K, Bickmore T. Social support agents for older adults: longitudinal affective computing in the home. J Multimodal User Interfaces 2014 Jun 18;9(1):79-88. [doi: 10.1007/s12193-014-0157-0]

57. Ring L, Utami D, Bickmore T. The right agent for the job? In: Intelligent Virtual Agents. Cham: Springer; 2014.

58. Ring L, Utami D, Olafsson S, Bickmore T. Increasing engagement with virtual agents using automatic camera motion. In: Intelligent Virtual Agents. Cham: Springer; 2016.

59. Sannon S, Stoll B, DiFranzo D, Jung M, Bazarova N. How personification and interactivity influence stress-related disclosures to conversational agents. In: Proceedings of the Computer Supported Cooperative Work and Social Computing. 2018 Presented at: CSCW '18: Computer Supported Cooperative Work and Social Computing; Nov 3-7, 2018; Jersey City, USA. [doi: $10.1145 / 3272973.3274076$ ]

60. Shamekhi A, Czerwinski M, Mark G, Novotny M, Bennett G. An exploratory study toward the preferred conversational style for compatible virtual agents. In: Intelligent Virtual Agents. Cham: Springer; 2016.

61. Smestad T, Volden F. Chatbot personalities matters: improving the user experience of chatbot interfaces. In: Internet Science. Cham: Springer; 2019.

62. Straßmann C, Krämer NC, Buschmeier H, Kopp S. Age-related differences in the evaluation of a virtual health agent's appearance and embodiment in a health-related interaction: experimental lab study. J Med Internet Res 2020 Apr 23;22(4):e13726 [FREE Full text] [doi: 10.2196/13726] [Medline: 32324146]

63. van Vugt HC, Konijn E, Hoorn J, Veldhuis J. Why fat interface characters are better e-health advisors. In: Proceedings of the 6th International Conference on Intelligent Virtual Agents, IVA 2006. 2006 Presented at: 6th International Conference on Intelligent Virtual Agents, IVA 2006; Aug 21-23, 2006; CA, United States. [doi: 10.1007/11821830 1]

64. Wissen A, Vinkers C, Halteren A. Developing a virtual coach for chronic patients: a user study on the impact of similarity, familiarity and realism. In: Proceedings of the 11th International Conference on Persuasive Technology - Volume 9638. 2016 Presented at: 11th International Conference on Persuasive Technology; Apr 5 - 7, 2016; Salzburg, Austria. [doi: 10.1007/978-3-319-31510-2 23]

65. Yin L, Bickmore T, Cortes D. The impact of linguistic and cultural congruity on persuasion by conversational agents. In: Intelligent Virtual Agents. Berlin, Heidelberg: Springer; 2010.

66. Zhang Z, Bickmore TW, Paasche-Orlow MK. Perceived organizational affiliation and its effects on patient trust: role modeling with embodied conversational agents. Patient Educ Couns 2017 Sep;100(9):1730-1737. [doi: 10.1016/j.pec.2017.03.017] [Medline: 28381330]

67. Zhou S, Bickmore T, Paasche-Orlow M, Jack B. Agent-user concordance and satisfaction with a virtual hospital discharge nurse. In: Intelligent Virtual Agents. Cham: Springer; 2014.

68. Zhou S, Zhang Z, Bickmore T. Adapting a persuasive conversational agent for the Chinese culture. In: Proceedings of the International Conference on Culture and Computing. 2017 Presented at: 2017 International Conference on Culture and Computing; Sep 10-12, 2017; Kyoto, Japan. [doi: 10.1109/culture.and.computing.2017.42]

69. Wang H, Kline JA, Jackson BE, Laureano-Phillips J, Robinson RD, Cowden CD, et al. Association between emergency physician self-reported empathy and patient satisfaction. PLoS One 2018 Sep 13;13(9):e0204113 [FREE Full text] [doi: 10.1371/journal.pone.0204113] [Medline: 30212564] 
70. Hojat M, Louis DZ, Maxwell K, Markham FW, Wender RC, Gonnella JS. A brief instrument to measure patients' overall satisfaction with primary care physicians. Fam Med 2011 Jun;43(6):412-417 [FREE Full text] [Medline: 21656396]

71. Derlega V, Winstead B, Greene K. Self-disclosure and starting a close relationship. In: Handbook of relationship initiation. New York, USA: Psychology Press; 2008.

72. Audet CT, Everall RD. Therapist self-disclosure and the therapeutic relationship: a phenomenological study from the client perspective. British J Guidance Counsel 2010 Aug;38(3):327-342. [doi: 10.1080/03069885.2010.482450]

73. Nass C, Steuer J, Tauber E. Computers are social actors. In: Proceedings of the SIGCHI Conference on Human Factors in Computing Systems. 1994 Presented at: CHI' 94: ACM Conference on Human Factors in Computing Systems; Apr 24-28, 1994; Boston, MA, USA. [doi: 10.1145/191666.191703]

74. Nass C, Moon Y. Machines and mindlessness: social responses to computers. J Social Isssues 2000 Jan;56(1):81-103. [doi: $\underline{10.1111 / 0022-4537.00153]}$

75. Mori M, MacDorman K, Kageki N. The uncanny valley [from the field]. IEEE Robot Automat Mag 2012 Jun;19(2):98-100. [doi: 10.1109/MRA.2012.2192811]

76. McTear MF. Spoken dialogue technology. ACM Comput Surv 2002 Mar;34(1):90-169. [doi: 10.1145/505282.505285]

77. Milne-Ives M, de Cock C, Lim E, Shehadeh MH, de Pennington N, Mole G, et al. The effectiveness of artificial intelligence conversational agents in health care: systematic review. J Med Internet Res 2020 Oct 22;22(10):e20346 [FREE Full text] [doi: 10.2196/20346] [Medline: $\underline{33090118]}$

\author{
Abbreviations \\ PRISMA-ScR: Preferred Reporting Items for Systematic Reviews and Meta-Analyses Extension for Scoping \\ Reviews

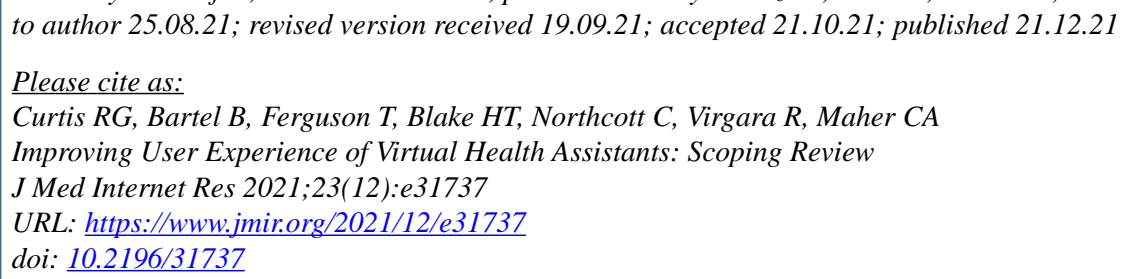

CRachel G Curtis, Bethany Bartel, Ty Ferguson, Henry T Blake, Celine Northcott, Rosa Virgara, Carol A Maher. Originally published in the Journal of Medical Internet Research (https://www.jmir.org), 21.12.2021. This is an open-access article distributed under the terms of the Creative Commons Attribution License (https://creativecommons.org/licenses/by/4.0/), which permits unrestricted use, distribution, and reproduction in any medium, provided the original work, first published in the Journal of Medical Internet Research, is properly cited. The complete bibliographic information, a link to the original publication on https://www.jmir.org/, as well as this copyright and license information must be included. 